\title{
Evaluating the Derivatives of Some Functions Using Maple
}

\author{
Chii-Huei Yu ${ }^{1, *}$, Bing-Huei Chen ${ }^{2}$ \\ ${ }^{1}$ Department of Management and Information, Nan Jeon University of Science and Technology, Tainan City, 73746, Taiwan \\ ${ }^{2}$ Department of Electrical Engineering, Nan Jeon University of Science and Technology, Tainan City, 73746, Taiwan \\ *Corresponding Author: chiihuei@mail.nju.edu.tw
}

Copyright $@ 2014$ Horizon Research Publishing All rights reserved.

\begin{abstract}
This article uses the mathematical software Maple for the auxiliary tool to study the differential problem of two types of functions. We can obtain the infinite series forms of any order derivatives of these two types of functions by using binomial series, Leibniz differential rule and differentiation term by term theorem, and hence greatly reduce the difficulty of calculating their higher order derivative values. On the other hand, we propose two examples to do calculation practically. The research methods adopted in this study involved finding solutions through manual calculations and verifying these solutions by using Maple. This type of research method not only allows the discovery of calculation errors, but also helps modify the original directions of thinking from manual and Maple calculations. For this reason, Maple provides insights and guidance regarding problem-solving methods.
\end{abstract}

Keywords Derivatives, Infinite Series Forms, Binomial Series, Leibniz Differential Rule, Differentiation Term By Term Theorem, Maple

\section{Introduction}

As information technology advances, whether computers can become comparable with human brains to perform abstract tasks, such as abstract art similar to the paintings of Picasso and musical compositions similar to those of Beethoven, is a natural question. Currently, this appears unattainable. In addition, whether computers can solve abstract and difficult mathematical problems and develop abstract mathematical theories such as those of mathematicians also appears unfeasible. Nevertheless, in seeking for alternatives, we can study what assistance mathematical software can provide. This study introduces how to conduct mathematical research using the mathematical software Maple. The main reasons of using Maple in this study are its simple instructions and ease of use, which enable beginners to learn the operating techniques in a short period. By employing the powerful computing capabilities of Maple, difficult problems can be easily solved. Even when Maple cannot determine the solution, problem-solving hints can be identified and inferred from the approximate values calculated and solutions to similar problems, as determined by Maple. For this reason, Maple can provide insights into scientific research. Inquiring through an online support system provided by Maple or browsing the Maple website (www.maplesoft.com) can facilitate further understanding of Maple and might provide unexpected insights. For the instructions and operations of Maple, [1-7] can be adopted as references.

In calculus and engineering mathematics courses, determining the $n$-th order derivative value $f^{(n)}(c)$ of a function $f(x)$ at $x=c$, in general, needs to go through two procedures: firstly finding the $n$-th order derivative $f^{(n)}(x)$ of $f(x)$, and then taking $x=c$ into $f^{(n)}(x)$. These two procedures will make us face with increasingly complex calculations when calculating higher order derivative values of a function (i.e. $n$ is large), and hence to obtain the answers by manual calculations is not easy. In this paper, we mainly study the differential problem of the following two types of functions

$$
\begin{aligned}
& f(x)=x^{p} e^{\lambda x} \cosh ^{q}(a x+b) \\
& g(x)=x^{p} e^{\lambda x} \sinh ^{q}(a x+b)
\end{aligned}
$$

where $\lambda, p, q, a, b$ are real numbers. We can obtain the infinite series forms of any order derivatives of these two types of functions by using binomial series, Leibniz differential rule, and differentiation term by term theorem; these are the major results of this study (i.e., Theorems 1 and 2), and hence greatly reduce the difficulty of calculating their higher order derivative values. The study of related differential problems can refer to [8-21]. On the other hand, we provide two examples to do calculation practically. The research methods adopted in this study involved finding solutions through manual calculations and verifying these solutions by using Maple. This type of research method not only allows the discovery of calculation errors, but also helps modify the original directions of thinking from manual and Maple calculations. Therefore, Maple provides 
insights and guidance regarding problem-solving methods.

\section{Main Results}

Firstly, we introduce two notations and three important theorems used in this study.

\subsection{Notation}

2.1.1. Suppose $r$ is any real number, $k$ is any positive integer. Define $(r)_{k}=r(r-1) \cdots(r-k+1)$, and $(r)_{0}=1$. 2.1.2. Assume $n, m$ are positive integers, and $m \leq n$. Define $\left(\begin{array}{l}n \\ m\end{array}\right)=\frac{n !}{m !(n-m) !}$, and $\left(\begin{array}{l}n \\ 0\end{array}\right)=1$.

\subsection{Binomial Series ([22, p244])}

$(1+y)^{r}=\sum_{k=0}^{\infty} \frac{(r)_{k}}{k !} y^{k}$, where $y, r$ are real numbers, $|y|<1$.

\subsection{Leibniz Differential Rule ([22, p121])}

Suppose $n$ is a non-negative integer, $f(x)$ and $g(x)$ are $n$-times differentiable functions. Then the $n$-th order derivative of the product function $f(x) \cdot g(x)$,

$$
(f \cdot g)^{(n)}=\sum_{m=0}^{n}\left(\begin{array}{c}
n \\
m
\end{array}\right) f^{(n-m)} g^{(m)}
$$

\subsection{Differentiation Term by Term Theorem ([22, p230])}

For all non-negative integers $k$, if the functions $g_{k}:(a, b) \rightarrow R$ satisfy the following three conditions : (i) there exists a point $x_{0} \in(a, b)$ such that $\sum_{k=0}^{\infty} g_{k}\left(x_{0}\right)$ is convergent, (ii) all functions $g_{k}(x)$ are differentiable on open interval $(a, b)$, (iii) $\sum_{k=0}^{\infty} \frac{d}{d x} g_{k}(x)$ is uniformly convergent on $(a, b)$. Then $\sum_{k=0}^{\infty} g_{k}(x)$ is uniformly convergent and differentiable on $(a, b)$. Moreover, its derivative $\frac{d}{d x} \sum_{k=0}^{\infty} g_{k}(x)=\sum_{k=0}^{\infty} \frac{d}{d x} g_{k}(x)$.

The following is the first result in this study, we determine the infinite series forms of any order derivatives of the function (1).

\subsection{Theorem 1}

Let $\lambda, p, q, a, b$ be real numbers, $n$ is a positive integer. Suppose the domain of the function

is $\left\{x \in R \mid x^{p}\right.$ exists, $\left.x \neq 0, a x+b \neq 0\right\}$.

$$
f(x)=x^{p} e^{\lambda x} \cosh ^{q}(a x+b)
$$

Case (A): If $a x+b>0$, and $x \neq 0$. Then the $n$-th order derivative of $f(x)$,

$$
f^{(n)}(x)=\frac{1}{2^{q}} e^{(\lambda+q a) x+q b} \sum_{m=0}^{n}\left(\begin{array}{l}
n \\
m
\end{array}\right)(p)_{n-m} x^{p-n+m} \cdot \sum_{k=0}^{\infty} \frac{(q)_{k}(\lambda+q a-2 a k)^{m}}{k !} e^{-2 k(a x+b)}
$$

Case (B): If $a x+b<0$, and $x \neq 0$. Then

$$
f^{(n)}(x)=\frac{1}{2^{q}} e^{(\lambda-q a) x-q b} \sum_{m=0}^{n}\left(\begin{array}{c}
n \\
m
\end{array}\right)(p)_{n-m} x^{p-n+m} \cdot \sum_{k=0}^{\infty} \frac{(q)_{k}(\lambda-q a+2 a k)^{m}}{k !} e^{2 k(a x+b)}
$$


Case (A): If $a x+b>0$, and $x \neq 0$. Because

$$
\begin{aligned}
& f(x)=x^{p} e^{\lambda x} \cosh ^{q}(a x+b) \\
= & x^{p} e^{\lambda x}\left[\frac{1}{2}\left(e^{a x+b}+e^{-(a x+b)}\right)\right]^{q} \\
= & \frac{1}{2^{q}} x^{p} e^{(\lambda+q a) x+q b}\left[1+e^{-2(a x+b)}\right]^{q} \\
= & \frac{1}{2^{q}} x^{p} e^{(\lambda+q a) x+q b} \cdot \sum_{k=0}^{\infty} \frac{(q)_{k}}{k !} e^{-2 k(a x+b)} \\
= & \frac{1}{2^{q}} x^{p} \cdot \sum_{k=0}^{\infty} \frac{(q)_{k}}{k !} e^{(\lambda+q a-2 a k) x+q b-2 b k}
\end{aligned}
$$

(By binomial series)

Differentiating $n$-times with respect to $x$ on both sides of (5), we obtain

$$
f^{(n)}(x)=\frac{1}{2^{q}} \sum_{m=0}^{n}\left(\begin{array}{c}
n \\
m
\end{array}\right)\left(x^{p}\right)^{(n-m)} \cdot\left(\sum_{k=0}^{\infty} \frac{(q)_{k}}{k !} e^{(\lambda+q a-2 a k) x+q b-2 b k}\right)^{(m)}
$$

(Using Leibniz differential rule)

$$
=\frac{1}{2^{q}} e^{(\lambda+q a) x+q b} \sum_{m=0}^{n}\left(\begin{array}{l}
n \\
m
\end{array}\right)(p)_{n-m} x^{p-n+m} \cdot \sum_{k=0}^{\infty} \frac{(q)_{k}(\lambda+q a-2 a k)^{m}}{k !} e^{-2 k(a x+b)}
$$

(By differentiation term by term theorem)

Case (B): If $a x+b<0$, and $x \neq 0$. Because

$$
\begin{aligned}
& f(x)=x^{p} e^{\lambda x}\left[\frac{1}{2}\left(e^{a x+b}+e^{-(a x+b)}\right)\right]^{q} \\
& =\frac{1}{2^{q}} x^{p} e^{(\lambda-q a) x-q b}\left[1+e^{2(a x+b)}\right]^{q} \\
& =\frac{1}{2^{q}} x^{p} e^{(\lambda-q a) x-q b} \cdot \sum_{k=0}^{\infty} \frac{(q)_{k}}{k !} e^{2 k(a x+b)} \\
& =\frac{1}{2^{q}} x^{p} \cdot \sum_{k=0}^{\infty} \frac{(q)_{k}}{k !} e^{(\lambda-q a+2 a k) x-q b+2 b k}
\end{aligned}
$$

(By binomial series)

Differentiating $n$-times with respect to $x$ on both sides of (6), we have

$$
\begin{gathered}
f^{(n)}(x)=\frac{1}{2^{q}} \sum_{m=0}^{n}\left(\begin{array}{c}
n \\
m
\end{array}\right)\left(x^{p}\right)^{(n-m)} \cdot\left(\sum_{k=0}^{\infty} \frac{(q)_{k}}{k !} e^{(\lambda-q a+2 a k) x-q b+2 b k}\right)^{(m)} \text { (By Leibniz differential rule) } \\
=\frac{1}{2^{q}} e^{(\lambda-q a) x-q b} \sum_{m=0}^{n}\left(\begin{array}{c}
n \\
m
\end{array}\right)(p)_{n-m} x^{p-n+m} \cdot \sum_{k=0}^{\infty} \frac{(q)_{k}(\lambda-q a+2 a k)^{m}}{k !} e^{2 k(a x+b)}
\end{gathered}
$$

(Using differentiation term by term theorem)

q.e.d.

The following is the second major result in this paper, we obtain the infinite series forms of any order derivatives of the function (2).

\subsection{Theorem 2}


Let the assumptions be the same as Theorem 1. Suppose the domain of the function

is $\left\{x \in R \mid x^{p}\right.$ exists, $\left.x \neq 0, a x+b \neq 0\right\}$.

$$
g(x)=x^{p} e^{\lambda x} \sinh ^{q}(a x+b)
$$

Case (A): If $a x+b>0$, and $x \neq 0$. Then the $n$-th order derivative of $g(x)$,

$$
g^{(n)}(x)=\frac{1}{2^{q}} e^{(\lambda+q a) x+q b} \sum_{m=0}^{n}\left(\begin{array}{l}
n \\
m
\end{array}\right)(p)_{n-m} x^{p-n+m} \cdot \sum_{k=0}^{\infty} \frac{(-1)^{k}(q)_{k}(\lambda+q a-2 a k)^{m}}{k !} e^{-2 k(a x+b)}
$$

Case (B): If $a x+b<0, x \neq 0$ and $(-1)^{q}$ exists. Then

$$
g^{(n)}(x)=\frac{(-1)^{q}}{2^{q}} e^{(\lambda-q a) x-q b} \sum_{m=0}^{n}\left(\begin{array}{l}
n \\
m
\end{array}\right)(p)_{n-m} x^{p-n+m} \cdot \sum_{k=0}^{\infty} \frac{(-1)^{k}(q)_{k}(\lambda-q a+2 a k)^{m}}{k !} e^{2 k(a x+b)}
$$

\subsubsection{Proof}

Case (A): If $a x+b>0$, and $x \neq 0$. Because

$$
\begin{aligned}
& g(x)=x^{p} e^{\lambda x} \sinh ^{q}(a x+b) \\
= & x^{p} e^{\lambda x}\left[\frac{1}{2}\left(e^{a x+b}-e^{-(a x+b)}\right)\right]^{q} \\
= & \frac{1}{2^{q}} x^{p} e^{(\lambda+q a) x+q b}\left[1-e^{-2(a x+b)}\right]^{q} \\
= & \frac{1}{2^{q}} x^{p} e^{(\lambda+q a) x+q b} \cdot \sum_{k=0}^{\infty} \frac{(-1)^{k}(q)_{k}}{k !} e^{-2 k(a x+b)} \\
= & \frac{1}{2^{q}} x^{p} \cdot \sum_{k=0}^{\infty} \frac{(-1)^{k}(q)_{k}}{k !} e^{(\lambda+q a-2 a k) x+q b-2 b k}
\end{aligned}
$$

(By binomial series)

Differentiating $n$-times with respect to $x$ on both sides of (9), we obtain

$$
\begin{gathered}
g^{(n)}(x)=\frac{1}{2^{q}} \sum_{m=0}^{n}\left(\begin{array}{c}
n \\
m
\end{array}\right)\left(x^{p}\right)^{(n-m)} \cdot\left(\sum_{k=0}^{\infty} \frac{(-1)^{k}(q)_{k}}{k !} e^{(\lambda+q a-2 a k) x+q b-2 b k}\right)^{(m)} \text { (By Leibniz differential rule) } \\
=\frac{1}{2^{q}} e^{(\lambda+q a) x+q b} \sum_{m=0}^{n}\left(\begin{array}{l}
n \\
m
\end{array}\right)(p)_{n-m} x^{p-n+m} \cdot \sum_{k=0}^{\infty} \frac{(-1)^{k}(q)_{k}(\lambda+q a-2 a k)^{m}}{k !} e^{-2 k(a x+b)}
\end{gathered}
$$

(Using differentiation term by term theorem)

Case (B): If $a x+b<0, \quad x \neq 0$ and $(-1)^{q}$ exists. Because

$$
\begin{aligned}
& g(x)=x^{p} e^{\lambda x}\left[\frac{1}{2}\left(e^{a x+b}-e^{-(a x+b)}\right)\right]^{q} \\
= & \frac{(-1)^{q}}{2^{q}} x^{p} e^{(\lambda-q a) x-q b}\left[1-e^{2(a x+b)}\right]^{q} \\
= & \frac{(-1)^{q}}{2^{q}} x^{p} e^{(\lambda-q a) x-q b} \cdot \sum_{k=0}^{\infty} \frac{(-1)^{k}(q)_{k}}{k !} e^{2 k(a x+b)} \\
= & \frac{(-1)^{q}}{2^{q}} x^{p} \cdot \sum_{k=0}^{\infty} \frac{(-1)^{k}(q)_{k}}{k !} e^{(\lambda-q a+2 a k) x-q b+2 b k}
\end{aligned}
$$

(By binomial series)

Differentiating $n$-times with respect to $x$ on both sides of (10), we have 


$$
\begin{aligned}
g^{(n)}(x) & =\frac{(-1)^{q}}{2^{q}} \sum_{m=0}^{n}\left(\begin{array}{l}
n \\
m
\end{array}\right)\left(x^{p}\right)^{(n-m)} \cdot\left(\sum_{k=0}^{\infty} \frac{(-1)^{k}(q)_{k}}{k !} e^{(\lambda-q a+2 a k) x-q b+2 b k}\right)^{(m)} \text { (By Leibniz differential rule) } \\
& =\frac{(-1)^{q}}{2^{q}} e^{(\lambda-q a) x-q b} \sum_{m=0}^{n}\left(\begin{array}{l}
n \\
m
\end{array}\right)(p)_{n-m} x^{p-n+m} \cdot \sum_{k=0}^{\infty} \frac{(-1)^{k}(q)_{k}(\lambda-q a+2 a k)^{m}}{k !} e^{2 k(a x+b)}
\end{aligned}
$$

(Using differentiation term by term theorem)

\section{Examples}

In the following, for the differential problem of the two types of functions in this study, we propose two examples and use Theorems 1, 2 to determine the infinite series forms of any order derivatives and some higher order derivative values of these functions. On the other hand, we employ Maple to calculate the approximations of these higher order derivative values and their solutions for verifying our answers.

\subsection{Example 1}

Suppose the domain of the function

$$
f(x)=x^{5 / 4} e^{6 x} \cosh ^{7 / 9}(2 x-4)
$$

is $\{x \in R \mid x>0, x \neq 2\}$.

3.1.1. If $x>2$. Then by Case (A) of Theorem 1, we obtain any $n$-th order derivative of $f(x)$,

$$
f^{(n)}(x)=\frac{1}{2^{7 / 9}} e^{\frac{68}{9} x-\frac{28}{9}} \sum_{m=0}^{n}\left(\begin{array}{l}
n \\
m
\end{array}\right)\left(\frac{5}{4}\right)_{n-m} x^{\frac{5}{4}-n+m} \cdot \sum_{k=0}^{\infty} \frac{\left(\frac{7}{9}\right)_{k}\left(\frac{68}{9}-4 k\right)^{m}}{k !} e^{-2 k(2 x-4)}
$$

Thus, we can determine the 13-th order derivative value of $f(x)$ at $x=3$,

$$
f^{(13)}(3)=\frac{1}{2^{7 / 9}} \cdot e^{\frac{176}{9}} \sum_{m=0}^{13}\left(\begin{array}{l}
13 \\
m
\end{array}\right)\left(\frac{5}{4}\right)_{13-m} 3^{\frac{-47}{4}+m} \cdot \sum_{k=0}^{\infty} \frac{\left(\frac{7}{9}\right)_{k}\left(\frac{68}{9}-4 k\right)^{m}}{k !} e^{-4 k}
$$

We use Maple to verify the correctness of (13) as follows:

$>\mathrm{f}:=\mathrm{x}->\mathrm{x}^{\wedge}(5 / 4) * \exp (6 * \mathrm{x}) *(\cosh (2 * \mathrm{x}-4))^{\wedge}(7 / 9)$;

$$
f:=x \rightarrow x^{5 / 4} \mathrm{e}^{6 x} \cosh (2 x-4)^{7 / 9}
$$

>evalf((D@@13)(f)(3),24);

$$
3.29604215167772942326529 \cdot 10^{20}
$$

$>\operatorname{evalf}\left(1 / 2^{\wedge}(7 / 9) * \exp (176 / 9) * \operatorname{sum}\left(13 ! /(\mathrm{m} ! *(13-\mathrm{m}) !) * \operatorname{product}(5 / 4-\mathrm{j}, \mathrm{j}=0 . .(12-\mathrm{m}))^{*} 3^{\wedge}(-47 / 4+\mathrm{m}) * \operatorname{sum}(\operatorname{product}(7 / 9-\mathrm{i}, \mathrm{i}=0 . .(\mathrm{k}-1)\right.\right.$ )$^{*}(68 / 9-4 * \mathrm{k})^{\wedge} \mathrm{m} / \mathrm{k} ! * \exp (-4 * \mathrm{k}), \mathrm{k}=0 .$. infinity $\left.\left.), \mathrm{m}=0 . .13\right), 24\right)$;

$$
3.29604215167772942326542 \cdot 10^{20}
$$

3.1.2. If $0<x<2$. By Case (B) of Theorem 1, we have any $n$-th order derivative of $f(x)$,

$$
f^{(n)}(x)=\frac{1}{2^{7 / 9}} e^{\frac{40}{9} x+\frac{28}{9}} \sum_{m=0}^{n}\left(\begin{array}{c}
n \\
m
\end{array}\right)\left(\frac{5}{4}\right)_{n-m} x^{\frac{5}{4}-n+m} \cdot \sum_{k=0}^{\infty} \frac{\left(\frac{7}{9}\right)_{k}\left(\frac{40}{9}+4 k\right)^{m}}{k !} e^{2 k(2 x-4)}
$$

Hence, we can evaluate the 15-th order derivative value of $f(x)$ at $x=1 / 2$,

$$
f^{(15)}\left(\frac{1}{2}\right)=\frac{1}{2^{7 / 9}} \cdot e^{\frac{48}{9}} \sum_{m=0}^{15}\left(\begin{array}{c}
15 \\
m
\end{array}\right)\left(\frac{5}{4}\right)_{15-m}\left(\frac{1}{2}\right)^{\frac{-55}{4}+m} \cdot \sum_{k=0}^{\infty} \frac{\left(\frac{7}{9}\right)_{k}\left(\frac{40}{9}+4 k\right)^{m}}{k !} e^{-6 k}
$$


Using Maple to verify the correctness of (15) as follows:

>evalf((D@@15)(f)(1/2),28);

$$
-5.3539044369458440384800701 \cdot 10^{13}
$$

$>\operatorname{evalf}\left(1 / 2^{\wedge}(7 / 9) * \exp (48 / 9) * \operatorname{sum}\left(15 ! /(\mathrm{m} ! *(15-\mathrm{m}) !)^{*} \operatorname{product}(5 / 4-\mathrm{j}, \mathrm{j}=0 . .(14-\mathrm{m}))^{*}(1 / 2)^{\wedge}(-55 / 4+\mathrm{m}) * \operatorname{sum}(\operatorname{product}(7 / 9-\mathrm{i}, \mathrm{i}=0 . .(\mathrm{k}-\right.\right.$ $1))^{*}(40 / 9+4 * \mathrm{k})^{\wedge} \mathrm{m} / \mathrm{k}$ ! $\exp (-6 * \mathrm{k}), \mathrm{k}=0$..infinity )$, \mathrm{m}=0 . .15), 26)$;

$$
-5.3539044369458440384800620 \cdot 10^{13}
$$

\subsection{Example 2}

Suppose the domain of the function

$$
g(x)=x^{16} e^{7 x} \sinh ^{4 / 3}(5 x+2)
$$

is $\left\{x \in R \mid x \neq 0,-\frac{2}{5}\right\}$.

3.2.1. If $x>-\frac{2}{5}$ and $x \neq 0$. Using Case (A) of Theorem 2, we can determine any $n$-th order derivative of $g(x)$,

$$
g^{(n)}(x)=\frac{1}{2^{4 / 3}} e^{\frac{41}{3} x+\frac{8}{3}} \sum_{m=0}^{n}\left(\begin{array}{l}
n \\
m
\end{array}\right)(16)_{n-m} x^{16-n+m} \cdot \sum_{k=0}^{\infty} \frac{(-1)^{k}\left(\frac{4}{3}\right)_{k}\left(\frac{41}{3}-10 k\right)^{m}}{k !} e^{-2 k(5 x+2)}
$$

Therefore, we obtain the 11-th order derivative value of $g(x)$ at $x=2$,

$$
g^{(11)}(2)=\frac{1}{2^{4 / 3}} \cdot e^{30} \sum_{m=0}^{11}\left(\begin{array}{l}
11 \\
m
\end{array}\right)(16)_{11-m} 2^{5+m} \cdot \sum_{k=0}^{\infty} \frac{(-1)^{k}\left(\frac{4}{3}\right)_{k}\left(\frac{41}{3}-10 k\right)^{m}}{k !} e^{-24 k}
$$

Verifying the correctness of (18) as follows:

$>\mathrm{g}:=\mathrm{x}->\mathrm{x}^{\wedge} 16^{*} \exp \left(7^{*} \mathrm{x}\right)^{*}\left(\left(\sinh \left(5^{*} \mathrm{x}+2\right)\right)^{\wedge} 4\right)^{\wedge}(1 / 3)$

$$
g:=x \rightarrow x^{16} \mathrm{e}^{7 x}\left(\sinh (5 x+2)^{4}\right)^{1 / 3}
$$

>evalf((D@@11)(g)(2),20);

$$
8.8339837832923512964 \cdot 10^{31}
$$

$>\operatorname{evalf}\left(1 / 2^{\wedge}(4 / 3) * \exp (30)^{*} \operatorname{sum}\left(11 ! /(\mathrm{m} ! *(11-\mathrm{m}) !) * \operatorname{product}(16-\mathrm{j}, \mathrm{j}=0 . .(10-\mathrm{m}))^{*} 2^{\wedge}(5+\mathrm{m}) * \operatorname{sum}\left((-1)^{\wedge} \mathrm{k}^{*} \operatorname{product}(4 / 3-\mathrm{i}, \mathrm{i}=0 . .(\mathrm{k}-1))\right.\right.\right.$ $*(41 / 3-10 * \mathrm{k})^{\wedge} \mathrm{m} / \mathrm{k} ! * \exp (-24 * \mathrm{k}), \mathrm{k}=0$. infinity $\left.\left.), \mathrm{m}=0 . .11\right), 20\right)$;

$$
8.8339837832923512972 \cdot 10^{31}
$$

3.2.2. If $x<-\frac{2}{5}$. By Case (B) of Theorem 2, we obtain any $n$-th order derivative of $g(x)$,

$$
g^{(n)}(x)=\frac{1}{2^{4 / 3}} e^{\frac{1}{3} x-\frac{8}{3}} \sum_{m=0}^{n}\left(\begin{array}{l}
n \\
m
\end{array}\right)(16)_{n-m} x^{16-n+m} \cdot \sum_{k=0}^{\infty} \frac{(-1)^{k}\left(\frac{4}{3}\right)_{k}\left(\frac{1}{3}+10 k\right)^{m}}{k !} e^{2 k(5 x+2)}
$$

Hence, we can determine the 14-th order derivative value of $g(x)$ at $x=-3$,

$$
g^{(14)}(-3)=\frac{1}{2^{4 / 3}} e^{\frac{-11}{3}} \sum_{m=0}^{14}\left(\begin{array}{l}
14 \\
m
\end{array}\right)(16)_{14-m}(-3)^{2+m} \cdot \sum_{k=0}^{\infty} \frac{(-1)^{k}\left(\frac{4}{3}\right)_{k}\left(\frac{1}{3}+10 k\right)^{m}}{k !} e^{-26 k}
$$

Also, we use Maple to verify the correctness of (20). 
>evalf((D@@14)(g)(-3),45);

$$
-3.083713394599021055518 \cdot 10^{10}
$$

$>\operatorname{evalf}\left(1 / 2^{\wedge}(4 / 3) * \exp (-11 / 3) * \operatorname{sum}\left(14 ! /(\mathrm{m} ! *(14-\mathrm{m}) !) * \operatorname{product}(16-\mathrm{j}, \mathrm{j}=0 . .(13-\mathrm{m}))^{*}(-3)^{\wedge}(2+\mathrm{m})^{*} \operatorname{sum}\left((-1)^{\wedge} \mathrm{k}^{*} \operatorname{product}(4 / 3-\mathrm{i}, \mathrm{i}=0\right.\right.\right.$. $(\mathrm{k}-1))^{*}(1 / 3+10 * \mathrm{k})^{\wedge} \mathrm{m} / \mathrm{k} ! * \exp (-26 * \mathrm{k}), \mathrm{k}=0$. .infinity $\left.\left.), \mathrm{m}=0 . .14\right), 26\right)$;

$$
-3.083713394599021055525 \cdot 10^{10}
$$

\section{Conclusion}

In this paper, we provide a new technique to evaluate the derivatives of some functions, we hope this technique can be applied to solve another differential problems. On the other hand, we know the binomial series, the Leibniz differential rule, and the differentiation term by term theorem play significant roles in the theoretical inferences of this study. In fact, the applications of these theorems are extensive, and can be used to easily solve many difficult problems; we endeavor to conduct further studies on related applications. In addition, Maple also plays a vital assistive role in problem-solving. In the future, we will extend the research topic to other calculus and engineering mathematics problems and solve these problems by using Maple. These results will be used as teaching materials for Maple on education and research to enhance the connotations of calculus and engineering mathematics.

\section{REFERENCES}

[1] M. L. Abell and J. P. Braselton, Maple by Example, 3rd ed., New York: Elsevier Academic Press, 2005.

[2] F. Garvan, The Maple Book, London: Chapman \& Hall/CRC, 2001.

[3] R. J. Stroeker and J. F. Kaashoek, Discovering Mathematics with Maple : An Interactive Exploration for Mathematicians, Engineers and Econometricians, Basel: Birkhauser Verlag, 1999.

[4] J. S. Robertson, Engineering Mathematics with Maple, New York: McGraw-Hill, 1996.

[5] C. Tocci and S. G. Adams, Applied Maple for Engineers and Scientists, Boston: Artech House, 1996.

[6] D. Richards, Advanced Mathematical Methods with Maple, New York: Cambridge University Press, 2002.

[7] C. T. J. Dodson and E. A. Gonzalez, Experiments in Mathematics Using Maple, New York: Springer-Verlag, 1995.

[8] M. Wagner, A. Walther, and B. J. Schaefer, On the efficient computation of high-order derivatives for implicitly defined functions, Computer Physics Communications, Vol. 181, Issue. 4, pp. 756-764, 2010.
[9] A. Griewank and A. Walther, Evaluating Derivatives: Principles and Techniques of Algorithmic Differentiation, 2nd ed., SIAM, Philadelphia, 2008.

[10] M. A. Patterson, M. Weinstein, and A. V. Rao, An efficient overloaded method for computing derivatives of mathematical functions in MATLAB, ACM Transactions on Mathematical Software (TOMS), Vol. 39, Issue. 3, 2013.

[11] C. -H. Yu, Using Maple to evaluate the derivatives of some functions, International Journal of Research in Computer Applications and Robotics, Vol. 1, Issue. 4, pp. 23-31, 2013.

[12] C. -H. Yu, A study on the differential problems using Maple, International Journal of Computer Science and Mobile Computing, Vol. 2, Issue. 7, pp. 7-12, 2013.

[13] C. -H. Yu, Evaluating the derivatives of two types of functions, International Journal of Computer Science and Mobile Computing, Vol. 2, Issue. 7, pp. 108-113, 2013.

[14] C. -H. Yu, A study on some differential problems with Maple, Proceedings of the 6th IEEE/International Conference on Advanced Infocomm Technology, No. 00291, 2013.

[15] C. -H. Yu, Application of Maple on solving some differential problems, Proceedings of IIE Asian Conference 2013, Vol. 1, pp. 585-592, 2013.

[16] C. -H. Yu, The differential problem of two types of functions, International Journal of Computer Science and Mobile Computing, Vol. 2, Issue. 7, pp. 137-145, 2013.

[17] C. -H. Yu, A Study on the differential problem, International Journal of Research in Aeronautical and Mechanical Engineering, Vol. 1, Issue. 3, pp. 52-57, 2013.

[18] C. -H. Yu, The derivatives of some functions, International Journal of Research in Information Technology, Vol. 1, Issue. 8, pp. 15-23, 2013.

[19] C. - H. Yu, Evaluating the derivatives of trigonometric functions with Maple, International Journal of Research in Computer Applications and Robotics, Vol. 1, Issue. 4, pp. 23-28, 2013.

[20] C. - H. Yu, The differential problem of some functions, International Journal of Computer Science and Mobile Applications, Vol. 1, Issue. 1, pp. 31-38, 2013.

[21] C. - H. Yu, The differential problem of four types of functions, Journal of Kang-Ning, vol. 15, pp. 51-63, 2013.

[22] T. M. Apostol, Mathematical Analysis, 2nd ed., Boston: Addison-Wesley, 1975. 